\title{
Effectiveness of cognitive training for Chinese elderly in Hong Kong
}

\author{
This article was published in the following Dove Press journal: \\ Clinical Interventions in Aging \\ 16 February 2013 \\ Number of times this article has been viewed
}

\section{Timothy Kwok ${ }^{1,2}$ \\ Anita Wong ${ }^{3}$ \\ Grace Chan ${ }^{4}$ \\ YY Shiu ${ }^{3}$ \\ Ko-Chuen Lam² \\ Daniel Young ${ }^{2}$ \\ Daniel WH Ho ${ }^{2}$ \\ Florence $\mathrm{Ho}^{2}$}

'Department of Medicine and Therapeutics, The Chinese University of Hong Kong, Hong Kong, People's Republic of China; 'Jockey Club Centre for Positive Ageing, Shatin, Hong Kong, People's Republic of China; ${ }^{3}$ The Hong Kong Chinese Women's Club Madam Wong Chan Sook Ying Memorial Care and Attention Home for the Aged, Hong Kong, People's Republic of China;

${ }^{4}$ The Hong Kong Council of Social Service, Hong Kong, People's Republic of China
Correspondence: Timothy Kwok Department of Medicine and Therapeutics, The Chinese University of Hong Kong, Hong Kong, People's Republic of China

Tel +852 26323145

Fax +852 26373852

Email tkwok@cuhk.edu.hk
Abstract: In Hong Kong, the evidence for cognitive-training programs in fighting against memory complaints is lacking. This study aimed to evaluate the effectiveness of the Active Mind cognitive-training program in improving the cognitive function and quality of life (QoL) for local community-dwelling Chinese older adults. A total of 200 subjects were recruited from 20 different district elderly community centers (DECCs). Centers were randomly assigned into either the intervention group or control group. The intervention group underwent eight 1-hour sessions of cognitive training, while the control group were included in the usual group activities provided by the DECCs. Standardized neuropsychological tests (the Chinese version of Mattis Dementia Rating Scale [CDRS] and the Cantonese version of the Mini-Mental State Examination) and the QoL questionnaire SF12 were used to assess participants' cognitive function and QoL before and after the trial. A total of 176 subjects completed the study. The intervention group showed greater improvement in the cognitive function measured by total CDRS score (treatment: $12.24 \pm 11.57$ vs control: $4.37 \pm 7.99 ; P<0.001$ ) and QoL measured by total SF12 score (treatment: $7.82 \pm 13.19$ vs control: $3.18 \pm 11.61 ; P=0.014$ ). Subjects with lower education level were associated with better cognitive response to the cognitive-training program. The current findings indicated that the Active Mind cognitive-training program was effective in improving the cognitive function and QoL for community-dwelling Chinese older adults in Hong Kong.

Keywords: cognitive training, cognitive stimulation, memory, older adults, effectiveness

\section{Introduction}

Elderly adults usually exhibit decline in memory and other cognitive abilities. This age-related cognitive decline is believed to be part of the normal aging process. ${ }^{1-3}$ However, the boundaries between normal aging and pathological cognitive loss are unclear, given that normal aging can progress to mild cognitive impairment or even dementia. ${ }^{4}$ As the number of older adults who are at risk of developing dementia will continue to increase with the aging population, effective preventive strategies for general cognitive decline are highly desirable. ${ }^{5}$

A large number of studies have demonstrated the role of cognitive training in slowing the age-related decline process, and in maintaining and enhancing the cognitive ability of older adults. ${ }^{6-11}$ A strong effect size for the impact of cognitive training on healthy older adults has been demonstrated in seven randomized controlled trials. ${ }^{5}$ It was also noted that the training effect is usually domain-specific. ${ }^{3,12}$ Ball et al found that intensive domain-specific training (eg, information-processing speed and reasoning) improved the cognitive abilities of older adults. ${ }^{13}$ Participation in activities that 
have a cognitive component is also found to be associated with reduced risk of developing cognitive impairment. ${ }^{14-16}$ Furthermore, previous memory-training programs were shown to improve perceived healthiness. For example, it was found that older adults who received an active cognitivetraining program were less likely to exhibit decline in healthrelated quality of life (QoL) than the controls. ${ }^{17}$

The cultural sensitivity of cognitive training should also be considered when applied to the Chinese population. Differences in linguistic property, cultural, and educational level may affect the consequence of training. For instance, Chinese older adults may not be able to understand the mnemonic strategies originally designed for the Western population. Therefore, training design should be culturally specific and the effectiveness of useful cognitive-training strategies for foreign populations may not be necessarily the same for local populations.

In Hong Kong, the evidence for the efficacy of cognitivetraining programs on memory complaint is lacking. This study aimed at evaluating the effectiveness of the Active Mind cognitive-training program in improving cognitive function and QoL on local community-dwelling Chinese older adults (aged 60 years or above). It was hypothesized that cognitive-training programs could improve cognitive function and enhance QoL for the subjects.

\section{Material and methods}

\section{Participants}

Two hundred participants were recruited through district elderly community centers (DECCs) in Hong Kong and the inclusion criteria were: (1) aged $\geq 60$ years, (2) scored 23 or above in the Mini-Mental State Examination (MMSE), (3) had not received any cognitive training in the past 3 months, (4) independent mobility, (5) no medical or psychiatric history associated with cognitive impairment (such as dementia, stroke, or traumatic brain injuries), and (6) was aware of memory difficulty or being noted by their close kin of memory loss yet the change was not severe enough to interfere with their daily living performance.

\section{Sample size}

The changes in the Mattis Dementia Rating Scale (DRS) score, which was the primary outcome measure of this study, were used to estimate the sample size required. Based on a pilot study conducted earlier, a mean improvement of 5 points in DRS score was found $(\mathrm{N}=15)$ in a cognitivetraining group. With an estimated power of $75 \%$ and alpha level of 0.05 , a total sample of 180 was required to detect an effect size of 0.2 . Taking a $10 \%$ possible attrition rate into account, the number was further rounded up to 200 so that there would be 100 participants in each group.

\section{Assessment tools}

Three assessment tools were used in the study. The Chinese version of the Mattis Dementia Rating Scale (CDRS) examines the level of cognitive functioning of our participants in five domains, namely attention, initiation/perseveration, construction, conceptualization, and memory. ${ }^{18}$ The maximum score of the unadjusted scale is 144 , with a Cronbach's alpha of 0.89 . Good psychometric properties were observed in both the original DRS and the Chinese version. ${ }^{19,20}$ In order to allow for direct comparisons, the raw total score and subscores of CDRS were adjusted according to participant's age and education, using the formulae recommended previously. ${ }^{19}$ The maximum total score of CDRS after adjustment can be higher than 144 .

The Cantonese version of the Mini-Mental State Examination (MMSE) assesses general cognitive status. It was translated and validated using the Hong Kong Chinese population. ${ }^{21}$ The total score is 30 . Cronbach's alpha is 0.86 . It has excellent clinical utilities in screening general cognitive decline.

The 12-item Short-Form Health Survey (SF12) in Chinese measures the QoL of the participants, with two foci: physical well-being and psychological well-being. ${ }^{22}$ The scale has been shown to be valid for use in the Chinese population in Hong Kong.

\section{Procedure}

This study was a joint effort between the Jockey Club Centre for Positive Ageing and the Hong Kong Council of Social Service (HKCSS). It aimed to evaluate the effectiveness of a cognitive-training program (Active Mind) targeted on general Chinese older adults living in the local community. Active Mind is a local cognitive-training program, with specific elements and modifications for adapting cultural differences in the local Chinese society. Particularly, Chinese surnames and photos of Chinese people are used in the naming module. Photos of Chinese items (eg, dim sum, Chinese-style calendar, teapot, etc) are used in the perception module. Chinese idioms are used in the executive functioning module. Traditional Chinese festivals (eg, lunar New Year, dragon boat festival, midautumn festival, etc) and corresponding signature items (eg, red pockets, dumplings, moon cakes, etc) are used in the imagery memory-training module. Popular Chinese songs and sound tracks of local 
events are used in the hearing memory-training module. Localized landmarks, scenic spots and transportation are used in the community-understanding module. And last but not least, Chinese names and popular local food are used in the self-completed exercises in the self-learning toolkit for the participant's easy understanding.

Data collection was conducted between September 2010 and March 2011. Subjects were recruited via the DECCs in Hong Kong. Out of 93 existing DECCs at that time, 44 centers agreed to participate in the study. Twenty were randomly selected. They were then randomly allocated at center level either into the intervention group or control group. Each center recruited 10 participants to join the study. There were 100 participants in either the treatment group or the control group. Informed consent was obtained and this study was approved by the ethics committee of the Chinese University of Hong Kong. The study complied with the tenets of the Declaration of Helsinki.

During the intervention period, in addition to usual group activities, the intervention group received eight Active Mind training sessions ( 1 hour per session, once a week for 8 weeks) administrated by either a social worker or an occupational therapist of each center. The Active Mind training program included education on memory deterioration and dementia, attention training, verbal fluency and association, mnemonics, method of loci, environmental awareness, lifestyle redesign, and memory aids. Homework was assigned after each lesson to reinforce learning and practice in daily life. Alternatively, the control group only received usual group activities provided by the participating DECCs, such as outings, monthly gathering, daily news reports and discussion. All activities (both intervention and control groups) were carried out in groups of about 10 people.

Associated diseases (eg, hypertension, diabetes, heart disease, stroke, psychiatric disease, Parkinson's disease, sleep problem, depression, etc) of the subjects were recorded. Besides obtaining demographic data, standardized neuropsychological tests (CDRS and CMMSE) and a QoL questionnaire (SF12) were used to assess the participants' cognitive abilities and QoL at baseline (the intervention period started immediately after the assessments) and immediately after the intervention period for both groups by trained research assistants, who were blinded to the group assignment.

\section{Statistical analysis}

SPSS statistical software (version 16.0; IBM, Armonk, NY, USA) was used for data analysis. Data were processed to obtain group mean value (M) and standard deviation (SD) or count $(\mathrm{N})$ and percentage (\%) where appropriate. The changes in cognitive abilities (CDRS and MMSE) and well-being (SF12) of participants (by determining the difference between baseline and after intervention period) in the treatment group and control group were compared to evaluate the effectiveness of the Active Mind program. Comparisons were performed by nonparametric Mann-Whitney $U$ tests and correlations were tested by nonparametric Spearman's correlation coefficient.

\section{Results}

A total of 200 participants were recruited. However, six of them did not meet the inclusion criteria and were excluded. After the study was completed, 176 subjects (90.7\%) remained and finished all the assessments (Figure 1).

\section{Baseline characteristics}

Table 1 shows the baseline characteristics of the participants. The mean age was 75.41 years with the majority being female (85.2\%) with only 3.56 years of education on average, which was relatively low. The majority were married (39.8\%) or widowed (54.5\%). 64.2\% were not living alone and they had on average one person to accompany them. Almost all of the subjects (94.9\%) did not have a maid and most (80.7\%) were not subsidized by Comprehensive Social Security Assistance (CSSA). Their mean MMSE score was 25.92 with SD of 2.30. The intervention and control groups showed no difference, except that the intervention group was relatively older (intervention, $77.41 \pm 6.75$ years vs control, $73.50 \pm 7.35$ years; $P=0.001$ ) and had relatively lower educational level (intervention, $2.92 \pm 3.36$ years vs control, $4.17 \pm 4.05 ; P=0.058$ ), and a higher proportion of intervention group subjects were subsidized by CSSA (intervention, $26.7 \%$ vs control, $12.2 \% ; P=0.015$ ). There was no significant difference in the frequencies of associated diseases between groups.

\section{Effectiveness of the Active Mind program as evaluated by outcome measures}

Table 2 summarizes the outcome measures of the participants. The difference between changes in cognitive status measured by total CDRS (comparing baseline and after intervention period) between intervention and control groups was highly significant (intervention, $12.24 \pm 11.57$ vs control, $4.37 \pm 7.99 ; P<0.001)$. Differences could also be detected in all the five subdomains (attention, initiation/perseveration, construction, conceptualization, and memory), with conceptualization being the most marked (treatment, $5.65 \pm 5.70 \mathrm{vs}$ control, $2.09 \pm 4.62 ; P<0.001)$. 


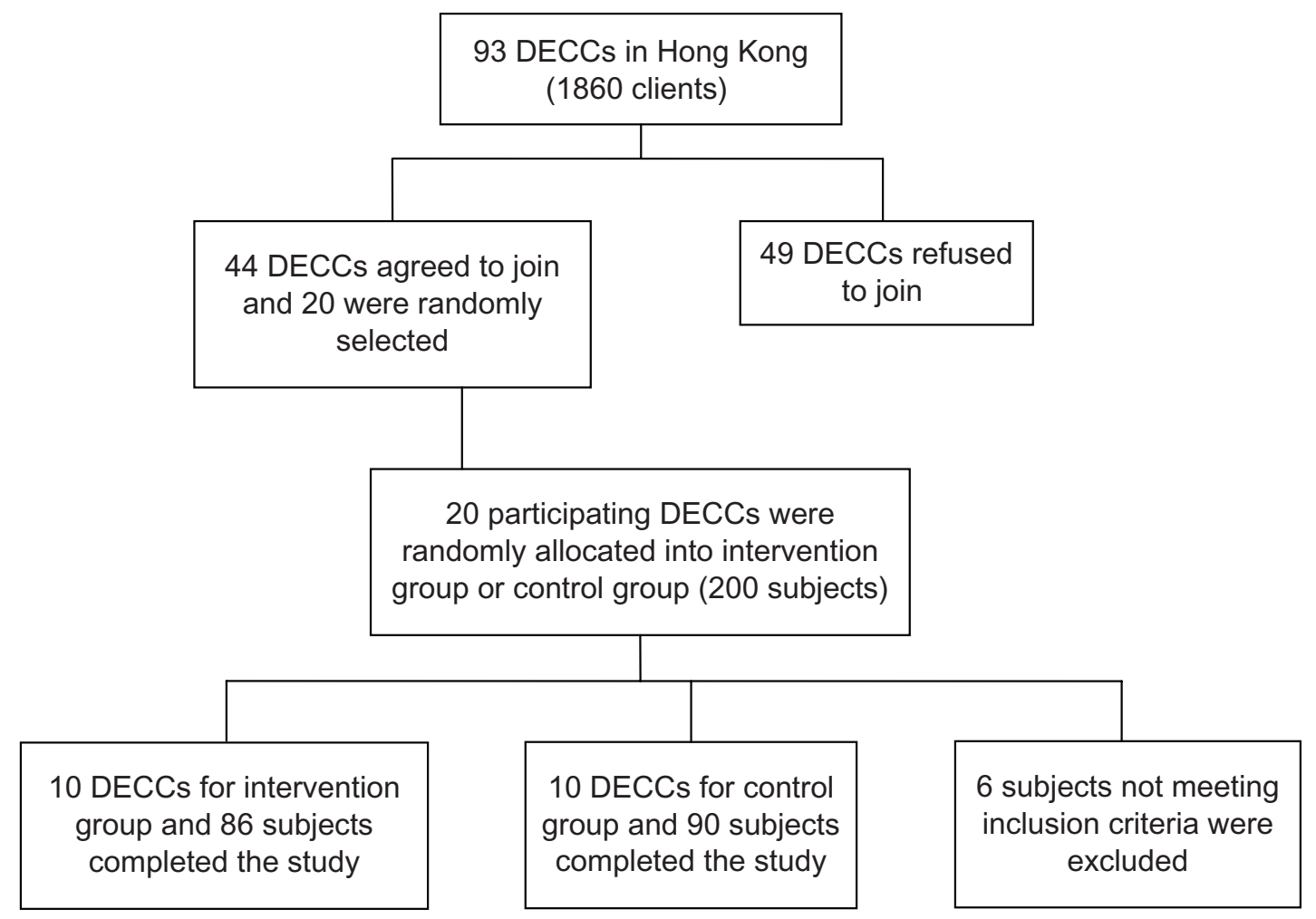

Figure I Procedure for subject recruitment.

Abbreviation: DECCs, district elderly community centers.

Table I Baseline characteristics of participants

\begin{tabular}{|c|c|c|c|c|}
\hline & \multicolumn{3}{|c|}{$M(S D) / N(\%)$} & \multirow[t]{2}{*}{$P$ value } \\
\hline & $\begin{array}{l}\text { Overall } \\
(N=176)\end{array}$ & $\begin{array}{l}\text { Intervention group } \\
(\mathbf{N}=\mathbf{8 6})\end{array}$ & $\begin{array}{l}\text { Control group } \\
(\mathrm{N}=90)\end{array}$ & \\
\hline Age & $75.4 I(7.3 I)$ & $77.4 I(6.75)$ & $73.50(7.35)$ & 0.001 \\
\hline Sex & & & & 0.469 \\
\hline Male & $26(14.8 \%)$ & II (I2.8\%) & $15(16.7 \%)$ & \\
\hline Female & I50 (85.2\%) & 75 (87.2\%) & 75 (83.3\%) & \\
\hline Education in years & $3.56(3.77)$ & $2.92(3.36)$ & $4.17(4.05)$ & 0.058 \\
\hline Marital status & & & & 0.628 \\
\hline Single & $6(3.4 \%)$ & $4(4.7 \%)$ & $2(2.2 \%)$ & \\
\hline Married & $70(39.8 \%)$ & $37(43.0 \%)$ & $33(36.7 \%)$ & \\
\hline Remarried & I (0.6\%) & $0(0 \%)$ & I (I.I\%) & \\
\hline Divorced & 2 (I.I\%) & I (I.2\%) & I (I.I\%) & \\
\hline Separated & I (0.6\%) & $0(0 \%)$ & I (I.I\%) & \\
\hline Widowed & 96 (54.5\%) & 44 (5।.2\%) & 52 (57.8\%) & \\
\hline Living arrangement & & & & 0.946 \\
\hline Alone & $63(35.8 \%)$ & $31(36 \%)$ & $32(35.6 \%)$ & \\
\hline Not alone & II3 (64.2\%) & 55 (64\%) & $58(64.4 \%)$ & \\
\hline Number of people living together (including self) & $1.98(1.03)$ & $1.88(0.90)$ & $2.18(1.13)$ & 0.391 \\
\hline Maid & & & & 0.357 \\
\hline Full time & $7(4.0 \%)$ & $3(3.5 \%)$ & $4(4.4 \%)$ & \\
\hline Part time & $2(1.1 \%)$ & $0(0 \%)$ & $2(2.2 \%)$ & \\
\hline Without maid & $167(94.9 \%)$ & $83(96.5 \%)$ & $84(93.3 \%)$ & \\
\hline Comprehensive Social Security Assistance (CSSA) & & & & 0.015 \\
\hline With CSSA & $34(19.3 \%)$ & $23(26.7 \%)$ & II (I2.2\%) & \\
\hline Without CSSA & $142(80.7 \%)$ & $63(73.3 \%)$ & $79(87.8 \%)$ & \\
\hline CMMSE & $25.92(2.30)$ & $25.74(2.27)$ & $26.09(2.33)$ & 0.322 \\
\hline
\end{tabular}

Abbreviations: CMMSE, Cantonese version of the Mini-Mental State Examination; M, mean value; SD, standard deviation. 
Table 2 Outcome measures of participants

\begin{tabular}{|c|c|c|c|c|c|c|c|}
\hline & \multicolumn{3}{|c|}{ Intervention group $(\mathbf{N}=\mathbf{8 6})$} & \multicolumn{3}{|c|}{ Control group $(\mathrm{N}=90)$} & \multirow[t]{2}{*}{$P$ value } \\
\hline & $\begin{array}{l}\text { Pretest } \\
\text { (M/SD) }\end{array}$ & $\begin{array}{l}\text { Posttest } \\
\text { (M/SD) }\end{array}$ & $\begin{array}{l}\text { Difference } \\
(\Delta M / S D)\end{array}$ & $\begin{array}{l}\text { Pretest } \\
\text { (M/SD) }\end{array}$ & $\begin{array}{l}\text { Posttest } \\
\text { (M/SD) }\end{array}$ & $\begin{array}{l}\text { Difference } \\
(\Delta M / S D)\end{array}$ & \\
\hline Total adjusted CDRS & I45.68 (|4.42) & $157.92(8.19)$ & 12.24 ( $\mid 1.57)$ & $152.80(10.73)$ & $157.17(8.23)$ & 4.37 (7.99) & $<0.001$ \\
\hline Attention & $33.11(2.82)$ & $34.64(1.88)$ & $1.52(3.03)$ & $34.00(2.84)$ & $34.56(\mathrm{I} .8 \mathrm{I})$ & $0.56(2.87)$ & 0.003 \\
\hline Initiation/perseveration & $43.88(6.03)$ & $46.20(4.59)$ & $2.33(5.07)$ & $45.76(4.84)$ & $46.39(3.95)$ & $0.63(4.55)$ & 0.013 \\
\hline Construction & $5.17(1.32)$ & $5.38(1.23)$ & $0.21(0.93)$ & $5.53(0.95)$ & $5.30(1.25)$ & $-0.23(1.17)$ & 0.028 \\
\hline Conceptualization & $27.56(5.80)$ & $33.21(2.83)$ & $5.65(5.70)$ & $31.62(3.92)$ & $33.71(3.42)$ & $2.09(4.62)$ & $<0.001$ \\
\hline Memory & $29.45(3.69)$ & $31.7 \mathrm{I}(2.67)$ & $2.26(3.03)$ & $30.06(3.52)$ & $31.38(2.59)$ & $1.78(2.94)$ & 0.015 \\
\hline Total SFI2 & $88.62(13.88)$ & $96.44(13.67)$ & $7.82(13.19)$ & $94.24(14.66)$ & $97.42(14.25)$ & $3.18(11.61)$ & 0.014 \\
\hline SFI2-physical & $38.95(10.29)$ & 39.23 (1 I.28) & $0.28(10.45)$ & $42.76(10.03)$ & $42.85(9.23)$ & $0.09(9.62)$ & 0.736 \\
\hline SFI2-mental & 49.66 (II.54) & $57.20(9.23)$ & $7.54(10.88)$ & 51.48 (II.14) & $54.56(9.02)$ & $3.08(10.16)$ & 0.005 \\
\hline
\end{tabular}

Abbreviations: CDRS, Chinese version of Mattis Dementia Rating Scale; $M$, mean value; SD, standard deviation; $\Delta M$, mean difference.

Similarly, for the QoL measured by SF12, the difference between treatment and control groups was found to be significant (treatment, $7.82 \pm 13.19$ vs control, $3.18 \pm 11.61$; $P=0.014)$. The total SF12 can be divided into physical and mental health subscores. Compared with the total score, both treatment and control groups showed improvement after intervention, but the change was more prominent in the treatment group. The difference in mental health subscore was statistically significant (treatment, $7.54 \pm 10.88$ vs control, $3.08 \pm 10.16 ; P=0.005)$ while that for the physical health subscale was not (treatment, $0.28 \pm 10.45$ vs control, $0.09 \pm 9.62 ; P=0.736)$.

\section{Variables related to cognitive improvement after the Active Mind program}

Considering only the treatment group subjects, higher education was negatively correlated with the improvement in total CDRS score (Spearman's correlation coefficient $=-0.279$; $P=0.009)$, CDRS attention subscore (Spearman's correlation coefficient $=-0.218 ; P=0.044)$ and CDRS conceptualization subscore (Spearman's correlation coefficient $=-0.263$; $P=0.014)$. It suggested that those with higher education levels tended to have less improvement from the Active Mind program. Specifically, by comparing literate and illiterate subjects, those who were illiterate had better improvement in CDRS overall score (illiterate, $18.96 \pm 13.48$ vs literate, $9.79 \pm 9.81 ; P=0.004)$, CDRS attention subscore (illiterate, $3.30 \pm 3.90$ vs literate, $0.87 \pm 2.37 ; P=0.003)$ and CDRS conceptualization subscore (illiterate, $8.87 \pm 6.77$ vs literate, $4.47 \pm 4.81 ; P=0.003)$. Therefore, older adults with lower education level or who were illiterate were suggested to have better improvement in cognitive ability after receiving the Active Mind program.

\section{Discussion}

The above findings confirmed that after attending the Active Mind cognitive-training program, the treatment group showed better improvement in cognitive function and QoL than the control group who only participated in the usual group activities. This study provides evidence supporting the effectiveness of cognitive-training program for communitydwelling Chinese elderly adults.

The subjects' characteristics were consistent with those attending other DECCs for older adults in Hong Kong. In order to target for the population of general Chinese elderly adults without apparent cognitive impairment, those who were known to have dementia or diseases leading to cognitive impairment were excluded. The results are therefore applicable to general community-dwelling Chinese older adults of good cognitive status. In this study, subjects with lower education level were more likely to benefit from the Active Mind program. This could be due to the ceiling effect of the cognitive-training program and more challenging tasks should be given to subjects with higher education levels.

Many DECCs in Hong Kong are serving older adults with a similar profile. The Active Mind cognitive-training program is therefore suitable to be carried out at various DECCs in Hong Kong. Older adults can benefit from this program by visiting the DECCs in their neighborhood for an enjoyable social encounter with other elderly in their vicinity. As a nonpharmacological intervention for people with memory complaints, it might be economically efficient to scale up this cognitive-training intervention. As the Active Mind cognitive-training program has been shown to be effective in improving cognitive function and QoL for local Chinese older adults, this program should be further promoted so that more elderly can benefit. 
This study was limited by the fact that aspects of age, educational level, and status of CSSA for the intervention and control groups were different at baseline. This was likely to be due to cluster randomization as the randomization was performed at center level instead of individually. Besides, despite the finding that significantly better improvement has been shown by the current study, the Active Mind program was rather short, with only eight 1-hour sessions performed once a week. The contrast for treatment and control groups will likely be increased if the cognitive-training program can be lengthened. Nevertheless, with the evidence of the current study, further studies of larger scale, longer studying period, and other potentially beneficial aspects of cognitive training are warranted. Mood and general affectivity may have an effect on cognitive improvement by the Active Mind program. It was also a limitation that these potential factors were not taken into account in evaluating our program.

In future, longitudinal studies with a longer followup period should be considered to determine whether cognitive-training programs could generate long-time effects on Chinese older adults and whether such programs could delay the progression of dementia or prevent cognitive loss. This should be considered because the long-term effect of cognitive training has been proven in a longitudinal study. ${ }^{23}$ In that study, for which more than 2800 participants were involved, they found that the improvements in the trained cognitive abilities could last for five years after the initiation of the intervention. Measurement at more frequent time intervals may also be useful to keep track of the changes of cognitive ability and mood.

In summary, the current study confirmed the effectiveness of the Active Mind cognitive-training program in improving cognitive function and QoL for community-dwelling Chinese older adults in Hong Kong. Subjects with lower education level or who were illiterate were found to benefit from the program. As such a characteristic is commonly found among clients of DECCs in Hong Kong, the current study provides justification for implementing extensive cognitive-training programs as a routine center activity.

\section{Acknowledgments}

The Active Mind toolkit was developed by the Hong Kong Chinese Women's Club Madam Wong Chan Sook Ying Memorial Care and Attention Home for the Aged. This study was generously sponsored by the Hong Kong Council of Social Service and CLP Power Hong Kong Limited.

\section{Disclosure}

Authors report no conflicts of interest in this work.

\section{References}

1. Deary IJ, Corley J, Gow AJ, et al. Age-associated cognitive decline. Br Med Bull. 2009;92:135-152.

2. Gunstad J, Paul RH, Brickman AM, et al. Patterns of cognitive performance in middle-aged and older adults: A cluster analytic examination. J Geriatr Psychiatry Neurol. 2006;19(2):59-64.

3. Kramer AF, Willis SL. Enhancing the cognitive vitality of older adults. Curr Dir Psychol Sci. 2002;11(5):173-177.

4. Whalley LJ. Brain ageing and dementia: what makes the difference? Br J Psychiatry. 2002;181:369-371.

5. Valenzuela M, Sachdev P. Can cognitive exercise prevent the onset of dementia? Systematic review of randomized clinical trials with longitudinal follow-up. Am J Geriatr Psychiatry. 2009;17(3):179-187.

6. Bissig D, Lustig C. Who benefits from memory training? Psychol Sci. 2007;18(8):720-726.

7. Eckroth-Bucher M, Siberski J. Preserving cognition through an integrated cognitive stimulation and training program. Am J Alzheimers Dis Other Dement. 2009;24(3):234-245.

8. Hertzog C, Kramer AF, Wilson RS, Lindenberger U. Enrichment effects on adult cognitive development: can the functional capacity of older adults be preserved and enhanced? Psychol Sci Public Interest. 2008;9(1):1-65.

9. Rebok GW, Carlson MC, Langbaum JB. Training and maintaining memory abilities in healthy older adults: traditional and novel approaches. J Gerontol B Psychol Sci Soc Sci. 2007;62B(Special Issue I): $53-61$.

10. Salthouse TA. Mental exercise and mental aging: evaluating the validity of the "Use it or lose it" hypothesis. Perspect Psychol Sci. 2006;1(1): 68-87.

11. Spector A, Thorgrimsen L, Woods B, et al. Efficacy of an evidence-based cognitive stimulation therapy programme for people with dementia: randomised controlled trial. Br J Psychiatry. 2003;183:248-254.

12. Spector A, Orrell M, Woods B. Cognitive Stimulation Therapy (CST): effects on different areas of cognitive function for people with dementia. Int J Geriatr Psychiatry. 2010;25(12):1253-1258.

13. Ball K, Berch DB, Helmers KF, et al. Effects of cognitive training interventions with older adults: a randomized controlled trial. JAMA. 2002;288(18):2271-2281.

14. Fabrigoule C, Letenneur L, Dartigues JF, Zarrouk M, Commenges D, Barberger-Gateau P. Social and leisure activities and risk of dementia: a prospective longitudinal study. $J$ Am Geriatr Soc. 1995;43(5): 485-490.

15. Wang JY, Zhou DH, Li J, et al. Leisure activity and risk of cognitive impairment: the Chongqing aging study. Neurology. 2006;66(6): 911-913.

16. Wilson RS, Mendes De Leon CF, Barnes LL, et al. Participation in cognitively stimulating activities and risk of incident Alzheimer disease. JAMA. 2002;287(6):742-748.

17. La Rue A. Health brain aging: Role of cognitive reserve, cognitive stimulation, and cognitive exercise. Clin Geriatr Med. 2010;26:99-111.

18. Chan AS, Choi MK, Salmon DP. The effects of age, education, and gender on the Mattis Dementia Rating Scale performance of elderly Chinese and American individuals. J Gerontol B Psychol Sci Soc Sci. 2001;56B(6):356-363.

19. Chan AS, Choi A, Chiu H, Lam L. Clinical validity of the Chinese version of Mattis Dementia Rating Scale in differentiating dementia of Alzheimer's type in Hong Kong. J Int Neuropsychol Soc. 2003;9: $45-55$.

20. Smith GE, Ivnik RJ, Malec JF, Kokmen E, Tangalos E, Petersen RC. Psychometric properties of the Mattis Dementia Rating Scale. Assessment. 1994;1(2):123-131. 
21. Chiu HF, Lam LC, Chi I, et al. Prevalence of dementia in Chinese elderly in Hong Kong. Neurology. 1998;50(4):1002-1009.

22. Lam CL, Tse EY, Gandek B. Is the standard SF12 health survey valid and equivalent for a Chinese population? Qual Life Res. 2005;14: $539-547$.
23. Willis SL, Tennstedt SL, Marsiske M, et al. Long-term effects of cognitive training on everyday functional outcomes in older adults. JAMA. 2006;296(23):2805n-2814n.

Clinical Interventions in Aging

\section{Publish your work in this journal}

Clinical Interventions in Aging is an international, peer-reviewed journal focusing on evidence-based reports on the value or lack thereof of treatments intended to prevent or delay the onset of maladaptive correlates of aging in human beings. This journal is indexed on PubMed Central, MedLine, the American Chemical Society's 'Chemical Abstracts
Dovepress

Service' (CAS), Scopus and the Elsevier Bibliographic databases. The manuscript management system is completely online and includes a very quick and fair peer-review system, which is all easy to use. Visit $\mathrm{http}: / /$ www.dovepress.com/testimonials.php to read real quotes from published authors.

Submit your manuscript here: http://www.dovepress.com/clinical-interventions-in-aging-journal 